\title{
ARTÍCULOS
}

\section{CONTENIDOS CULTURALES DE GÉNERO EN LA RELIGIOSIDAD AIMARA DEL NORTE DE CHILE*}

\author{
CULTURAL CONTENTS OF GENDER IN THE AYMARA RELIGIOSITY OF NORTHERN \\ CHILE
}

\section{Vivian Gavilán ${ }^{1}$}

Universidad de Tarapacá

Recibido: 27 de septiembre de 2017; Aprobado: 24 de enero de 2018; Publicado online: 9 Abril 2019

Cómo citar este artículo / Citation: Gavilán, Vivian. 2019. «Contenidos culturales de género en la religiosidad aimara del norte de Chile». Disparidades 74(1): e009. doi: <https://doi.org/10.3989/dra.2019.01.009>.

RESUMEN: Los estudios de la religiosidad en comunidades aimara han informado sobre la importancia que adquieren los sistemas culturales de género en la organización de los rituales y en los sistemas clasificatorios que estructuran las representaciones del orden social. Considerando estos antecedentes se presentan aquí resultados de una investigación que se propuso explorar en los sujetos de culto y sus contextos. A través de entrevistas y observaciones etnográficas, aplicadas en dos comunidades del altiplano del norte chileno, me aproximo a las divinidades y sus relaciones para conocer las bases filosóficas del proceso de simbolización de los géneros. Comparo los datos acopiados en dos períodos de trabajo de campo (1982-1997 y 2007 y 2016 ). Los resultados informan sobre el devenir de los procesos coloniales de género, caracterizado por la imposición de la ideología judeocristiana. También muestran la lucha de los actores sociales por incluir saberes y creencias legadas por los antepasados. Las referencias al dominio sexual conducen a contradicciones con el sistema hegemónico. La heterogeneidad de las ideas y de las prácticas religiosas se manifiesta en campos semánticos que resisten amalgamarse. Pero, los sistemas escolar y de salud se unen a las iglesias para continuar evangelizando y reduciendo lo femenino a la maternidad.

PALABRAS CLAVE: Aimara; Religiosidad; Género; Sexualidades; Pueblos indígenas.

SUMMARY: Studies of religiosity in Southern Andean Aymara communities have reported on the importance of cultural systems of gender in the theoretical configuration of rituals. Considering these preceding studies, I present here the results of an investigation that intends to explore the "subjects of worship" and their contexts, given the role they play as key actors in local history. Through interviews and ethnographic observations, conducted in two communities of Northern Chile's high plateau, I have explored the divinities and their relationships to understand the philosophical bases of the process of gender symbolization. I compare the data collected in two periods of fieldwork (1982-1997 and 2007-2016). The results testify to the evolution of colonial processes of gender, characterized by the imposition of Judeo-Christian ideology. At the same time, they show how social actors struggle to include knowledge and beliefs acquired from ancestors. References to the sexual domain lead to contradictions with the hegemonic system. The heterogeneity of the ideas and religious practices is manifested in a superposition of gender traditions that resist amalgamation; however, the school and health systems join the churches in continuing to evangelize and reduce the feminine to motherhood.

KEYWORDS: Aymara; Religiosity; Gender; Sexualities; Indigenous Peoples.

Copyright: (C) 2019 CSIC. Este es un artículo de acceso abierto distribuido bajo los términos de la licencia de uso y distribución Creative Commons Reconocimiento 4.0 Internacional (CC BY 4.0).

* Este artículo forma parte del proyecto titulado: Estudio de las relaciones de género en las narrativas del pasado en la colectividad aimara del norte chileno, auspiciado por el Concurso Proyectos Mayores de Investigación Científica y Tecnológica Universidad de Tarapacá 2018. Forma parte, también, del trabajo de investigación que desarrolla la autora como doctoranda del Programa de Doctorado en Antropología y Comunicación del Departamento de Antropología, Filosofía y Trabajo Social, Universitat Rovira i Virgili, Catalunya, España.

1 Correo electrónico: viviangav@yahoo.com. ORCID iD: <https://orcid.org/0000-0002-6913-378X>. 


\section{INTRODUCCIÓN}

La religiosidad de los aimara es un campo de estudio fértil para el abordaje de la construcción simbólica del género. Las prácticas discursivas y las acciones ceremoniales se inscriben en un sistema de clasificaciones en el que predominan las ideas que tienen las personas que pertenecen a esta colectividad sobre la diferencia sexual.

A fines de la década de los setenta del siglo pasado Strathern (1979: 136) afirmó que el género podía ser comprendido como un conjunto de ideas que expresan las percepciones sobre el sexo y que no son principalmente unas reglas o normas, a pesar de que pueden tener un efecto normativo y que sirven para simbolizar un conjunto de otras relaciones sociales, aplicarse a otros dominios de la realidad social y para poner en mutua relación las diferencias de sexo. En estos años Ortner y Whitehead (1981) afirmaron que el género, entendido como símbolo, puede ser estudiado desde dos formas. De un lado, el desciframiento de la lógica interna y las relaciones estructurales entre los símbolos culturales; del otro, el análisis de las relaciones entre símbolos y significados y ciertos aspectos de las relaciones sociales. En esta perspectiva, las autoras sugieren que las estructuras de prestigio son fundamentales para la elaboración de los conceptos de sexo y género; ya que estas median entre los dominios del parentesco (moldeador de la concepción de hombres y mujeres) y las ideologías de género. Plantean que muchos aspectos de las concepciones particulares del género cobran sentido si se las interpreta como procedentes de la perspectiva de los actores, quienes actúan de acuerdo con las reglas y mecanismos que rigen la diferenciación del estatus en su sociedad.

Por otra parte y siguiendo a Lacan, Lamas (1996) sugiere que el género, como simbolización de la diferencia sexual, se construye culturalmente diferenciado en un conjunto de prácticas, ideas y discursos, entre los que se encuentran los de la religión. Se entiende que en cada cultura la oposición hombre/mujer pertenece a una trama de significaciones determinadas que puede expresarse en los registros simbólico, imaginario y real, por lo cual se debería desentrañar la red de interrelaciones e interacciones sociales desde la división simbólica de los sexos.

Concebir el género como un constructo simbólico permite poner la atención en el sistema de valoraciones, en cómo las diferentes sociedades piensan y sienten las diferencias genéricas y, al mismo tiempo, puede ser entendido como parte de un sistema cultural que los actores pueden manipular, interpretar, legitimar y reproducir los patrones que ordenan su mundo social (Collier y Rosaldo 1981: 313).

Los investigadores y las investigadoras del sur andino han expuesto cómo el sistema de género permea diferentes ámbitos de la vida social en general; y, en la religiosidad, en particular. Isbell (1997) indicó que un cuidadoso examen de la ritualidad andina nos ayudaría a comprender los esquemas de género en las diversas tradiciones culturales de la región, y que aún hoy es posible visibilizar la valoración cultural propia que estas comunidades asignan al sexo y a lo femenino como abstracción. Al hablar del lugar que ocupa el género en la estructura social y simbólica de las comunidades aimaras rurales de Bolivia, Spedding (1997: 59) propuso que era secundario; ya que al articularse con edad o parentesco puede tomar el segundo lugar y no siempre emerge como sexo binario; tampoco se puede presuponer la relación jerárquica entre lo masculino y lo femenino.

Si bien los estudios etnográficos de la religiosidad de los aimaras en la región de Tarapacá, zona en la que se centra este trabajo, no se han enfocado en la construcción simbólica del género, entregan importante información respecto de las representaciones de lo femenino y lo masculino. Una parte de estos ha tendido a poner énfasis en el actor para conocer categorías émicas que refieran a la cosmovisión indígena contemporánea (Grebe 1981; Martínez 1976). Otras investigaciones han optado por modelos interpretativos que postulan la existencia de una matriz cultural precolombina susceptible de ser reconstruida. Se tiende a identificar niveles de originalidad y niveles de sincretismo como modelos explicativos (Kessel 1992a, 1992b, 1993 y 2007; Guerrero 1990 у 1994).

Considerando estas afirmaciones me interesó indagar en los contenidos de las prácticas religiosas que aluden a las diferencias y semejanzas entre lo femenino y lo masculino y sus valoraciones en la colectividad aimara que reside en el norte chileno ${ }^{2}$. Al estudiar los símbolos de género, asumo que las representaciones de las diferencias sexuales se han corporizado en las personas y sus grupos sociales a

2 En 1998 se publicaron los resultados de investigación de la primera fase de trabajo. Aquí se amplían los antecedentes y se comparan los datos con los acopiados en los últimos años. 
través de los procesos históricos (Appadurai 2001). En este sentido, los elementos que componen las tradiciones culturales presentes en la colectividad aimara provienen de los procesos coloniales y neocoloniales regionales. Las tradiciones de los pueblos originarios no son culturas puras y las de los no indígenas en la región no son mestizas, son mixtas, hechas de continuidades y discontinuidades; además, estas últimas se ubican más en el orden temporal que en el orden espacial (Bastide, en Cuche 2004). Es decir, es importante considerar el carácter histórico de los símbolos de género (Canessa 1997).

Desde este marco referencial expongo aquí resultados de un estudio que se propuso comparar datos etnográficos recogidos en dos comunidades aimaras del altiplano chileno en las décadas de los ochenta y noventa con registros levantados en los últimos diez años (1982-1997 y 2007-2016). Desde un enfoque cualitativo puse atención en las prácticas vinculadas a la religiosidad de los miembros de estas colectividades, mediante el levantamiento de datos etnográficos en largas temporadas. Para el primer período, la información proviene de observaciones realizadas en fiestas patronales y carnavales y veintiséis entrevistas, semiestructuradas cuyas preguntas se orientaron a hablar de la fe y el bienestar de las personas y la familia, sobre las peticiones para las actividades productivas, para la salud y la suerte, término frecuentemente utilizado para referirse a los destinos personales. Se entrevistaron a once hombres entre los 35 y 81 años y a quince mujeres entre los 27 y 65 años de edad. Entre los hombres se entrevistaron a seis católicos, tres evangélicos y dos sin adscripción en la actualidad, aunque habían sido católicos y pentecostales. Entre las mujeres, ocho son católicas, cuatro evangélicas y tres sin adscripción. Para el segundo período, se utilizaron observaciones de fiestas patronales (San Juan de Cariquima y Santo Tomás de Isluga) y de carnavales de estas mismas comunidades y diez entrevistas abiertas; en estas se incluyeron cuatro mujeres y dos hombres que participaron de la investigación anterior y que se adscriben tanto a la iglesia católica como a la evangélica pentecostal; las cuatro personas restantes son mujeres y hombres jóvenes.

Los contextos históricos de ambos períodos son distintos. El siglo pasado se caracterizó por una gran presión de la sociedad nacional por homogenizar culturalmente a la población. Las prácticas religiosas se desplegaban con recato y con cierto temor a la represión histórica del catolicismo hegemónico y a la discriminación étnica. En las últimas décadas, caracterizadas por el reconocimiento de los pueblos originarios por parte del estado y nación chilenos, este temor ha disminuido notablemente. Más aún, el pasado se recupera para enfatizar la diferencia cultural en la sociedad nacional. Si bien en la segunda mitad del siglo pasado las personas adscritas a la religión evangélica y los/las jóvenes fueron reticentes a hablar sobre el tema, ya sea por prohibición, inhibición o desconocimiento, actualmente tanto los primeros como los segundos se refieren, con comodidad, a las prácticas religiosas que la costumbre indica y se resignifican en clave política en búsqueda de una nueva forma de integración o inclusión a la sociedad chilena.

\section{LA RELIGIOSIDAD AIMARA}

En general existe consenso en que la religiosidad actual de la población de origen indígena en el sur andino es producto de las diversas expresiones religiosas nativas que imperaron durante el período prehispánico, especialmente aquellas dirigidas por el Estado inka y la imposición del cristianismo agenciada por la Iglesia católica y protestante. Hay acuerdo también en que la evangelización no ha terminado y en que la colonización ha sido violenta y excluyente. El debate existe en torno a cómo calificar la combinación de diversas tradiciones. Algunos señalan que existiría una síntesis entre las creencias antiguas y el cristianismo. Se habla de integración para referirse a como la tradición indígena incorporó el Evangelio. Otros, en cambio, prefieren llamarlo sincretismo religioso debido a que se trataría de una mezcla o yuxtaposición de las creencias indígenas y cristianas, puesto que existiría el dualismo religión clandestina/religión oficial ${ }^{3}$.

Podríamos caracterizar a la religiosidad indígena, pues, como una lucha histórica por legitimidad y una batalla de ideologías, en la que la cristiana ha contado con mayores herramientas institucionales para imponerse y subalternizar a las tradiciones nativas. Los aimaras, sin embargo, como sujetos sociales han contado con elementos culturales propios para reflexionar y crear nuevas formas de pensar y configurar prácticas

3 Juliane Esch-Jakob (1994: 94) asegura que según sus estudios no se podría hablar de síntesis entre las dos religiones porque la esencia del Evangelio no pertenece al pensamiento indígena como eje central. 
a través de interpretaciones que les provee la historia vivida en sus comunidades. Como señala Isbell (1997: 300) la historia del género en los Andes es una batalla entre las ideologías andina y cristiana.

Ciertamente, las actuales relaciones de poder sitúan al cristianismo en una posición hegemónica y subordinan aquellas prácticas que se inscriben en el ámbito de lo que los aimaras llaman la costumbre, entendida esta como conjunto de actividades que, si bien hoy continúan otorgando las bases para la alteridad en la sociedad regional y nacional, se caracterizan por incluir ideas cristianas apropiadas desde la filosofía precristiana subalternizada.

Las transformaciones en la vida religiosa se manifiestan hoy en una gran heterogeneidad social y cultural en la que se encuentran los aimaras en Chile. Actualmente se adscriben a las Iglesias católica, evangélica pentecostal, evangélica nazarena, adventista, sabática del Séptimo Día, del Evangelio cuadrangular, metodistas pentecostales, testigos de Jehová. Con excepción de la primera, todas son adopciones que se fueron produciendo en la segunda mitad del siglo $X X$. Asistimos, también, a la no adscripción eclesiástica, aunque se adhieren al cristianismo y/o a lo que han llamado religiosidad andina.

En las comunidades altiplánicas del norte chileno es en los años ochenta del siglo pasado que se produjo una masiva conversión hacia la Iglesia evangélica pentecostal. Existió un gran fanatismo que descalificó todo acto relacionado con la costumbre. Aquellos que profesaron el culto protestante tendieron a dejar de practicar, de manera abrupta o paulatina, ceremonias y rituales que realizaban siendo católicos. Quienes se adscribieron al catolicismo continuaron con la vieja tradición de festividades del ciclo anual. Este siglo, sin embargo, se ha caracterizado por una mayor valoración de la diferencia; por lo tanto, la costumbre es relevada para marcar identidades étnicas que comenzaron a sobresalir en gran parte de la población vinculada a las comunidades rurales de Tarapacá.

En ambos períodos considerados se observan cambios generacionales. Las personas mayores con menor escolaridad son reconocidas por la comunidad por sus saberes y competencias para seguir la tradición de los antepasados. Las entrevistas confirman que las personas con mayor manejo de la lengua aimara y con poca escolaridad reflexionan y explican las relaciones entre las divinidades y comparten la lógica de los rituales. Al contrario, los jóvenes y sol- teros con mayor escolaridad afirman no saber y aplican contenidos aprendidos en la escuela o iglesias. No obstante, los dirigentes jóvenes de organizaciones que reivindican derechos a la diferencia cultural y que residen principalmente en las ciudades se esfuerzan por recuperar la memoria colectiva para su lucha política. En estos casos, los rituales y sus contextos experimentan modificaciones si las comparamos con la que realizaban las familias campesinas en la segunda mitad del siglo pasado.

Actualmente la mayoría de la población de valles y altiplano reside en las ciudades de la costa. Sin embargo, la movilidad y los procesos migratorios no han borrado las diferencias étnicas y la religiosidad contribuye a sustentarlas. En este sentido, el concepto de translocalización de la comunidad aimara hacia el espacio urbano adquiere relevancia. Gundermann y González (2008) sugieren que la colectividad aimara ha formado parte de un proceso de desruralización (por las emigraciones y movilidad), desagrarización (del patrón de ingresos), descampesinización (de la organización económica) y translocalización (de los patrones de relaciones sociales). Las relaciones con la comunidad rural son centrales para la vida en la ciudad, y las familias migrantes inciden en la localidad de manera decisiva tanto económica como políticamente. El vínculo con la comunidad de origen se expresa, también, en la importancia que adquieren las festividades como carnavales, fiestas patronales y los días de muertos.

En este contexto, las transformaciones socioeconómicas y socioculturales han profundizado la subalternización de los discursos y prácticas no cristianas. Pero, al mismo tiempo, persisten las representaciones de género allí donde la cultura cristiana hegemónica no dispone de contenidos. Me refiero a la sexualidad como dimensión central de la producción y reproducción de la vida y cuyos contenidos contribuyen a concebir la naturaleza de los géneros. Estos contenidos sustentan procesos de identidad/alteridad con la población que no se adscribe a los pueblos originarios.

\section{SUJETOS DE CULTO}

Las entrevistas aplicadas para el primer período considerado (1982-1997) dan cuenta de una gran flexibilidad en cuanto a la centralidad del culto por parte de los comuneros y comuneras. Las respuestas no fueron homogéneas respecto de la principal divinidad. Los católicos de mayor edad identifican su principal fe en 
sus uywiri (cerros o vertientes) ${ }^{4}$. Aparece Mama Candelaria u otro santo o santa, patrono/a de su localidad de origen. Entre los evangélicos, Dios Padre. En relación a las prácticas rituales, la mayoría identifica tradiciones familiares para expresar distinciones en sus formas. Esto incluye particularidades entre esposo-esposa, ya que la procedencia de las mujeres (de otras localidades) implica adherir a los uywiris de su familia paterna. La indagación no consideró temas tales como la convivencia del pentecostalismo y catolicismo, creencias cristianas y no cristianas. El foco se puso en describir a los sujetos de culto, ya sea del presente y/o del pasado (cf. Gavilán 1998).

A diferencia de lo planteado por Spedding (1997) para la zona rural de Bolivia las figuras divinas veneradas en estas comunidades, para los dos períodos considerados, muestran una clara referencia al sexo binario y una marcada unidad entre lo masculino y lo femenino. Tata Kollanta (Tata Dios o Inti) ocupa el primer lugar en el primero. Se trata de un referente de la más alta jerarquía. Su par, en el orden de lo femenino, es Pachamama (Virgen Tayka, Santa Tierra, Mama Virgin). Esta entidad se desdobla en MallkuT'alla, entidades masculino-femenina que habitan diferentes espacios territoriales y accidentes geográficos: los seres de los cerros, de las vertientes y de las pirkas que se convierten en Pachamama, y también denominados como uywiris o criadores y mantenedores. En un lugar de menor jerarquía se ubican las figuras del santoral católico, pero son resignificadas siguiendo las tradiciones nativas.

Las deidades reseñadas forman parte del universo o pacha en el que se distinguen: arajj pacha o mundo de arriba y aca pacha o este mundo. Ambos espacios están signados por uno de los géneros: arriba-masculino, abajo-femenino. Como veremos, las relaciones que se establecen entre ellos y ellas se pueden observar en los rituales. El mundo de adentro o manqha

4 El término uywiri alude a crianza. El diccionario de Pedro García (2002), miembro de la comunidad de Isluga, señala que la palabra uywa designa 'animal-ganado', reduciendo el término a la cría de animales. Bertonio, indica que uywaña denomina la crianza de las personas como de los animales. Martínez (1976: 37) califica a estos como aviadores, indica que Bravo Q'ari en aimara se refiere a la naturaleza temible y peligrosa de los lugares marcados con un fuerte grado de sacralidad; "lo poderoso es peligroso y aquí en Isluga los poderes de un aviador están casi siempre acompañados en proporción directa a los prestigios, de los cuales forma parte por los peligros que emanan de esta ambivalencia de lo sagrado". pacha, descrito por otros autores, no apareció, de manera explícita, en ninguna de las entrevistas; lo cual está en directa relación con la formulación de los protocolos de entrevistas, pero también con un ámbito que no es fácil abordar con los no aimara. En las comunidades de la zona altiplánica de la región de Tarapacá, Jutur Mallku y Jutur T'alla es la referencia más cercana a lo demoníaco y peligroso. Sin embargo, esto no es así en la zona que corresponde a la región de Arica y Parinacota, donde estas entidades son mencionadas con frecuencia. De todas maneras, es común que los mallkus y t'allas sean nominados como demonios, particularmente por los evangélicos.

\subsection{Kollantatata}

Inti (Dios-Sol) o Kollantatata es traducido por mujeres y hombres entrevistados como 'Dios Padre' ${ }^{5}$. Su masculinidad es asociada a la paternidad entendida como protector de los humanos. El nombre de kollanta alude a Señor Principal. El diccionario de Ludovico Bertonio (1984 [1612]: 50) indica que collana quiere decir 'excelente, prima cosa, collanancatha ser el primero, estar en primer lugar'. Por otra parte, la expresión aimara kollan auqui nos refiere a padre principal. Cumple la función de proteger del mal y líder del buen camino. Las expresiones "él nos pastea» o "él es nuestro pastorero" permiten comprender sus atributos y significados. Contiene la idea de cuidar con cariño, guiar por el camino correcto, velar por la vida, proteger de catástrofes y de peligros. Su lugar en el mundo lo expone la expresión usada por todos los entrevistados: «siempre tiene que ir por delante de toda ceremonia o pedimento. Es el primerísimo que ayuda a la gente. Vive en el cielo». Se recuerda con incienso y cuando está saliendo el sol, de rodillas en la puerta de la cocina. En todo momento, pero las abuelas lo hacían especialmente los días jueves y sábado.

Tata kollanta se relaciona con la primera luz del día. Un fragmento de la entrevista a un comunero de Cariquima nos facilita su comprensión:

- ¿Cuando hace la wilancha ${ }^{6}$, a quién se pide?

5 En aimara también recibe los nombres de tatita arajj $p a-$ cha, kollan auquista. En español, El Señor, Tata Dios, Jesucristo, Santísimo, Santo Rey.

6 Wilancha nombra el donar sangre (de los animales sacrificados) como ofrenda principal a los seres tutelares. Es el acto de ofrendar sangre (wila). 
-Al señor, ¿qué es el tata kollanta, que quiere decir alumbró, ve?, ¿floreció el corazón, ve? Esa es primera que hay que rocear, después mallku-t'alla, da tu bendición, yo te cumplí... ¿Por qué kollanta quiere decir 'cuando alumbró, que ya apenas que nos vio', ¿¿ve?

-¿Al Sol le están diciendo Tata...?

-Al Sol sí, claro, kollanta es Sol.

- ¿No dicen Inti, kollanta no más, pero ese el Tata Dios...

-Sí.

- ¿Cuál es el rezo en el momento de la wilancha al Sol?

- Tata kollanta, kollanta t'alla, uywirpa, ¿quiere decir 'mantiene todo', 'mantiene al ganado'?, uywiri mantiene al ganado, ¿'ve? Ahí.

- ¿Ese es uywiri?

-Sí, uywiri. Ese es el primer cristo que nos alumbra, esa es la antigua...

- ¿Dónde está el Inti?, ¿dónde está el kollanta?

- El Sol, en el cielo vive con gente.

- ¿Con quién vive kollanta en el cielo?

-No sé bien.

- ¿Está en el cielo no más?

-En el cielo no más.

- ¿Cómo pides al kollanta?

-Acordamos al Señor en castellano, kollanta en aimara.

- ¿Cuándo se recuerda al kollanta, al Inti?

- Cuando sale el sol, cuando uno se acuerda, cuando uno viaja o pasa cosas malas, se acuerda.

- ¿Puedes decir una oración completa en la wilancha?

- Juma dios yatista, juma uintista, juma utasista, jani walijanas, por castellano: 'Usted sabe bien cómo estoy, tú me cuidas de los malos, si estoy mal me echas en el agua', total, usted sabe (J. F. Hombre, 50 años, Cariquima, entrevista personal, [1990]).

Su equivalencia con Santísimo o Jesucristo es explicitado por otros entrevistados:

- ¿Cómo es el Inti?

-Inti es el Sol.

- ¿Dónde está Inti?

-El Inti está en el cielo, de ahí tenemos que rogar, porque ese es el nuestro Jesucristo que es el que nos da también.

- ¿Cómo se le pide al Inti?

-Con un poquito de puro [alcohol] y así se hace un sahumerio.

- ¿Cómo se le recuerda al Inti?

-Al Inti hay que pedirle bien, a veces le decimos

Santísimo, es Santísimo.

- ¿Tu sabes cómo se recuerda al Sol?

-El Inti, en la mañana cuando está saliendo sol siempre mi abuelita con incienso, creo que siempre estaba acordando abuela madre, con incienso cuando está saliendo solcito, Santísimo, hincadito en la puerta de la cocina sabe estar, pidiéndose pues. Siempre yo me acuerdo de eso.

- ¿Todos los días?

-No los días jueves y sábado, hay que hacerse siempre. Ese siempre yo me acuerdo de mi abuelita koIlantata, chorancha con... nayay uywir ..kollantata, se sabe estar pidiendo. Yo sé poco hablar aimara también, no sabemos nada también. Pa una parte los papás, las mamás como no le enseña a uno, no le enseña na. En castellano, le está diciendo «Padre Dios, cuídame hoy día, alúmbrame» (M. E. Mujer, 60 años, Cariquima, entrevista personal, [1990]).

Durante todo el año se le implora y en todos los rituales asociados a las fiestas propiciatorias de la agricultura y ganadería. Se pide ayuda para la salud, en momentos difíciles, etc. El incienso y el alcohol son los elementos rituales básicos para pedir a koIlanta. Otros comuneros dicen que para las peticiones a Dios no aplica la ofrenda de sangre de un animal (wilancha). Sin embargo, este ritual se realiza en todas las celebraciones importantes a la salida del sol, momento en el que se le implora y reza.

El lugar donde habita Tatita Dios y con quien es difuso. Solo en dos de los entrevistados aparece koIlanta t'alla ${ }^{7}$ o Señora principal. Las reflexiones dejan un vacío a la hora de describir la acompañante de Santísimo-Inti. Ninguno de los entrevistados/as mencionó a la Luna como objeto de culto. La existencia de una contraparte femenina supondría que, como en el caso de los uywiris, la pareja masculino-femenino es también válida en el mundo de arriba o arajj pacha. M. Esther Grebe, antropóloga que trabajó en las mismas comunidades que nos referimos aquí, informa sobre la partición Dios Parte y Uywir Parte. La primera sería habitada por Koyan Santísimo o Inti Sol, quien estaría casado con Phajsi Mama (Luna, Virgen tayka, Virgen María, identificada con Pachamama). Estos tendrían por hijos a las estrellas. Ella ubica a Pachamama en el taipi pacha (aka pacha o este mundo) como mediadora entre arajj pacha y manqha

7 Tristán Platt (1988: 407) indica que la palabra Ttalla en el vocabulario de Bertonio, expresaba la fertilidad abundante de las mujeres. El diccionario registra en la página 347 que la palabra Ttalla Ccapkhomi significaba 'reina mujer del señor principal del pueblo y las parientas cercanas de los mallkus'. En tanto que el diccionario de Pedro García (2002 [1997]), perteneciente a la comunidad de Isluga, incluye el término t'alla y lo define como 'esposa del cacique y reina'. 
pacha; pero no establece relaciones claras entre Inti y Pachamama (Grebe 1981).

La luna como referente femenino del mundo de arriba fue registrado por Tristan Platt en sus estudios sobre los macha, comunidad quechua de Bolivia. Como Grebe, ubica a la pareja divina Tata Inti = Padre Sol / Mama Killa = Madre Luna, en la mitad superior - Pata Parti Pacha (o dimensión superior = Hanan Pacha); mientras que dentro de la tierra se ubicaría Pachatata (Padre Tierra) Pachamama (Madre Tierra), en la mitad inferior o Ura Parti (o dimensión inferior = Ukhu Pacha). Esta última deidad recibiría también el nombre de virgen, pero en este grupo cultural se le asignaría mayor importancia a la fertilidad de la Madre de Dios que a su castidad (Platt 1980: 158). Asimismo, cada una de estas mitades contienen implícitamente referencias sexuales y en relación asimétrica (mayor o menor prestigio y poder). Sin embargo, las parejas ubicadas en la misma parcialidad, como es el caso de la Luna y Pachatata son figuras ambivalentes (femenino-masculino y masculino-femenino respectivamente), lo que implica que los macha pueden afirmar simultáneamente una unión entre los rayos fertilizantes del Sol y la tierra productiva: «el tata Inti es puramente masculino; la Pachamama puramente femenina» (Platt 1980: 160).

Como se mencionó antes, los entrevistados no mencionaron a la Luna como referente de culto y si bien el hábitat de Inti o Kollantata es arriba (cielo), está presente en el aca pacha (representada por Pachama$m a$ ) como entidad masculina que activa la fertilidad.

\subsection{PACHAMAMA-MALLKU-T'ALLA}

El análisis de los antecedentes respecto de Pachamama dio como resultado una figura de un alto estatus. Es fundamentalmente fértil, proveedora de alimentos y cobija la vida en su interior. Sus atributos aluden a un ser femenino y madre, contenedora de la vida: es dadora de productos agrícolas y de pastos para los animales. Está asociada también al dinero. Ella es la tierra y está viva, son los cerros y las chacras. El lugar del rito son justamente los cerros, los pukaras (montículos de piedras que marcan sitios que representan a antepasados) ${ }^{8}$, donde pasta el ganado y se

8 Pedro García (2002 [1997]: 66-67), comunero de Isluga, define la palabra pukara como lugar sagrado o sitio donde se hace una ceremonia. ubican los terrenos agrícolas. Se relaciona siempre a kollanta tata en las peticiones y en las fiestas patronales se le recuerda a través de la wilancha y de la acción de challar (ofrendar chicha en chuwa, collar con coca y kopal) ${ }^{9}$. Sus vinculaciones con Dios es difusa. La mayoría de las personas entrevistadas sugieren que Dios está primero y después hay que recordarse de las pachamamas, o sea los cerros, los lugares donde comen los animales.

En las ceremonias que se ofrenda a Pachamama se invocan a los uywiris: Mallku-T'alla. Jutur Mallku o Jutur T'alla, Inca Mallku Inca T'alla, etc. Los términos Mallku y T'alla nos remiten a una categoría de cargos de representación social, política y ritual de alto prestigio, asignada también a hombres y mujeres de la comunidad cuando asumían la condición de caciques. Reciben también el nombre de achachilas o abuelos. Viven en distintos lugares del territorio familiar y comunal. En las ceremonias adoptan nombres propios: María Cruzani (corresponde a Mama Wanapa, montaña que protege a la comunidad de Cariquima), Manuel Atora y Pancha Caranga (son las referencias del Tata Jachura, montaña de alta jerarquía en toda la región, pero protector de las familias de los valles altos de la cordillera occidental de los Andes que se ubica hoy en la región de Tarapacá). De esta manera, cerros, vertientes o pirkas se presentan siempre en términos de género: femenino-masculino. La presencia de la pareja puede estar contenida en un mismo lugar o pueden ubicarse en el territorio en forma separada.

Se representan como una pareja de comuneros vestidos a la usanza antigua. En las ceremonias agrícolas se visten talegas con semillas o bien piedras, con toda la indumentaria que usaron en el pasado. En los rituales propiciatorios de la agricultura, especialmente en cosechas, rendían culto a una imagen femenina, sin su pareja masculina. En este caso se visten costales de quinua o papa que representa la hermana quinua o hermana papa; las que pueden ser denominadas como mama, mas no como t'alla; marcando las jerarquías entre las entidades femeninas. Las mujeres entrevistadas argumentan que va solo la mujer porque cualquier producción es mujer.

9 Challar implica rociar alimento o bebida en la tierra. Chuwa son los recipientes que contienen chicha especialmente destinados a las divinidades. Kopal es una piedra volcánica que se se utiliza como incienso y como remedio para dolores estomacales y mareos. 
Se homologan a la humanidad: tienen sexo-género, son amantes o matrimonio, tienen nombres propios, tienen hijos, etc. En tanto seres personificados con capacidades sobrenaturales, dominan los espacios circundantes de la población determinando las actividades productivas, la salud y el bienestar de las personas; pueden dar vida y dar muerte. Su alimento preferido es la sangre, les gusta beber y compartir con sus devotos; resguardan el orden y la moral de los habitantes. Podemos ver que comúnmente los uywiris son representados como adultos y normalmente en pareja, en edad fértil y productiva.

Otra característica importante es su presencia nocturna o su regreso al tiempo presente en el período correspondiente a Todos los Santos y carnavales (tiempo de lluvias estivales, de primicias, fiesta del ganado y fiesta de la gente). Normalmente son convocados a la media noche, ceremonia semisecreta a la que asisten mujeres y hombres casados, quienes representan a las unidades domésticas, estancias y/o la comunidad.

Al mismo tiempo que son protectores, son proveedores de agua, pastos, alimentos y salud. Si no se les recuerda, considerando su estatus, suelen generar desordenes y caos en las personas, familia y/o comunidad. Esto se manifiesta en escasez de recursos, mal vivir y en enfermedades graves como kajkata o agarradura, fenómeno que se produce cuando los uywiri bravos les extraen el animu $u^{10}$ a las personas en momentos de hambre y enojo.

Estas características han sido ampliamente descritas. Bouysee-Cassagne y Harris (1987: 45) han señalado que para los agricultores los cerros son los ejes significativos del paisaje, y la mayor concentración de las fuerzas del mankha pacha. Son los más hambrientos entre todos. Las principales ofrendas rituales suelen ser para ellos. Por un lado hacen enfermar por su hambre, pero también pueden ser grandes curanderos.

\subsection{LOS SANTOS Y LAS SANTAS}

Siguiendo el orden colonial de la reducción de la población a pueblos, las comunidades históricas de Isluga y Cariquima se organizan en torno a un territorio cuyo pueblo central o marka incluye una iglesia

10 Componente esencial para el funcionamiento de los organismos vivos. Cf. Gavilán et al. (2011). con santos y santas venerados por todas las familias que pertenecen a la misma. En tanto que las estancias, localidades en las que la mayoría trabaja y reside, tienen iglesias con santas y santos de menor jerarquía cuyos devotos corresponden a unidades familiares menores.

Las representaciones de los santos y de las santas se asemejan a las parejas mallku-t'alla; aun cuando se observen diferencias. Estos no tienen el estatus de antepasados de las actuales generaciones, no son inkas, no son uywiris (criadores) o awatiris (pastores), no se celebran en los cerros, pirkas o vertientes. Sus ofrendas rituales no consideran chuwa ni colla. Las mesas rituales no se hacen en la piedra, ni en la tierra, sino en la mesa central de la plaza.

Las mujeres entrevistadas sugieren que cada santo o virgen posee un genio propio: temperamento bueno o temperamento malo. Pueden ser humildes, atropelladores o poco generosos. Algunos/as son más o menos celosos. Ello implica respeto para los ofrecimientos de sus devotos. Si no se les cumple lo prometido, castigan, trayendo enfermedades o mala suerte. Los santos son como mujer y hombre, no pueden celebrarse solos, ello significa que siempre tienen que salir a la procesión acompañados con sus compañeros de vivienda, es decir, con quienes comparten el altar en la iglesia. Aunque los comuneros dicen que si en alguna iglesia se encuentran solos/ as, se celebran con su compañera/o (Santo Tomás con Mamita Concepción o Santa Bárbara, Tatita San Felipe con Mamita Candelaria, etc.).

Al representarlos como personas se conciben como miembros de la localidad. Argumentan que viven en la iglesia como cualquier otro comunero. El día de la celebración, se les pide bendición (buena cosecha, que dé alimentos) para que den suerte en el comercio, para tener buena salud, por milagros. Los caracterizan como protectores, lo que equivalen a abogados. Es decir, los defienden de la mala suerte, de sequías, de atentados, etc.

Cada año se hace cargo una unidad familiar para celebrar su día, representada por un hombre y una mujer (esposo-esposa, hermana-hermano, madrehijo, padre-hija). Las entrevistas muestran que en los días de fiesta se revelan a través de los sueños para comunicarles que deben pasar el cargo de cacicazgo. Los santos para pasar el cargo de mallku y las santas a las mujeres para el cargo de t'alla. 
Hay una clara jerarquía entre los santos: de mayor a menor inclusión, de mayor a menor importancia: patronas/os de la unidad familiar (siblings), de estancias y de comunidad. Igual que mallkus y t'allas hay santos y vírgenes que abarcan más de una comunidad. Este es el caso de la Virgen de Copacabana y San Santiago de Yungullo, ambos en territorio boliviano.

La celebración dura por lo general tres días y sigue la misma estructura de las demás festividades: antevíspera, víspera, día grande, cacharpaya o despedida. En música predominan los instrumentos de viento (sikura y lichiguayos), aunque las bandas de viento y bandas electrónicas complementan durante todos los días de fiesta.

\section{LO MASCULINO Y LO FEMENINO EN LAS REPRESENTACIONES DE LAS DIVINIDADES}

Las ceremonias y rituales, especialmente aquellas que propician la agricultura y la ganadería, muestran las relaciones que los aimaras establecen entre las figuras divinas. El ritual de la siembra es descrita por dos mujeres de Isluga:

Hay que pedirse del señor su bendición para que viva y de bendición. Primero del Señor y después el virgen, wilanchamos, collamos y nombramos nuestros terrenos, nuestras chacras. Después Pindinchau, Pachamama virgen sipiti (inyecciones) o sea; sembramos hoyito para embarazar la tierra para que crezca planta indin... estamos echando con hojita, covando, challando para que esté en buena condición, o sea con voluntad. De esa forma sahumamos con incienso con kopala. Pal Señor siempre incienso pal Sol, porque hace orear todo. El Sol en primer lugar, botan el alcohol purito, pa pedirse. En primer lugar, por ejemplo dice que se tiene que inchune como dice esa es la mama de la quinua de la papa también. Eso, por ejemplo, estás embarazao, estas esperando, tú lo tienes en tu estómago, está sembrao, ya la criatura se parece de chiquito a grande, igual la planta también se siembra. Por eso decimos sirpat; así estamos challando así acordamos, virgen ya estamos diciendo plantita recogimos, ¿ंve? (M. F. y E. C. Mujeres 50 y 30 años, Isluga, entrevista, [1988]).

El ritual agrícola une lo masculino, representado en kollantatata, Inti, Cristo o Santísimo y ubicado en el arajj pacha o mundo de arriba con lo femenino, representado en Pachamama, figura que se desdobla en mallku-t'alla (masculino-femenino). La expresión embarazar la tierra nos sitúa en el dominio de la fecundación. Coherentemente, al consultar a mujeres y hombres aimaras respecto de la reproducción biológica humana ambos utilizaron la metáfora de la siembra. A la pregunta del papel de la mujer y del hombre en la fecundación la mujer dice: «o sea, por ejemplo, igual que sembrar el maíz en buena tierra es bueno el fruto, pero si no, no; yo creo que es más....es la mujer la que le da la fuerza a la guagua [bebé] porque está dentro del vientre de la madre». El hombre responde: «bueno, el padre aporta lo que es él, él es como la semilla del hijo y la madre lo que aporta la crianza de los nueve meses».

Esta misma argumentación, pero en otro sentido sugiere Alejandro Ortiz (1982) para los chanka de Moya, sur del Perú: la madre da el cuerpo, el padre la vida. Ella pone la materia y su forma, el padre el semen que es vida pura y despierta lo dormido. Gabriel Martínez (1976 y 1989) habla de la sexualidad como componente de los uywiris (mallku-t'alla) y como dimensión de la simbolización de la organización social dual en Isluga. Al describir la mesa ritual él propone que el Sol y el rayo (oculto en la figura de San Santiago) poseen un significado de fecundadores masculinos; mientras que la Luna se marca por su fertilidad. De este modo, lo masculino, representado en el arajj pacha, es fecundador de lo de abajo, representado como femenino en la figura de la matriz que contiene la vida en este mundo.

La persistencia de las parejas sexuales en el pensamiento andino es propuesta por Domingo Jiménez (citado en Albó y Layme 1992: 149): "Las deidades siempre han de ser pareja: Madre Tierra y Cumbre; Cumbre y Madre Tierra. Nosotros también nos casamos y somos pareja, así también son ellos».

Para la zona que aquí se estudia, Kessel (1992b: 211 y 2007) identifica "elementos culturales autóctonos y externos que se acomodarían en el panteón según el principio de tripartición». Ubica a Inti, Dios, Sol, junto a la Virgen María y a los santos en el mundo de arriba o arajj pacha. Si bien la deidad femenina se halla en arajj pacha como lo hace Grebe (1996), esta no es igual a Luna. Para este autor Pachamama se encontraría relacionada con otras dos figuras importantes: el Mallku o espíritu de las montañas y el Amaru, la serpiente que se asocia al agua y especialmente a la limpieza y apertura de los canales de riego. Propone que se trataría de un triple culto a la fertilidad que se centra en el agua como dadora de vida. Sugiere que a pesar del proceso de sincretismo 
que se ha producido entre Pachamama (llamada también virgina) y la Virgen María serían claramente distinguibles para los aimaras. Ello se expresaría tanto en los rituales como en su designación. Mientras esta última se ubica en el cielo, la deidad andina pertenecería al akapacha o este mundo. Sin embargo, para Kessel existiría similitud de funciones: ser madre universal y ser virgen; a las cuales no se les reconoce esposo ${ }^{11}$. Tienen que ver con la vida humana, pero mientras María solo dispone indirectamente de la fertilidad del campo y del ganado, Pachamama es madre de toda la flora y la fauna. Su maternidad es también diferente: esta última cuida con cariño maternal la fertilidad y la salud, la alimentación de la vida y el bienestar de sus hijos en la tierra, que la respetan; María, en cambio, fue representada por los misioneros como maternidad espiritual (Kessel 1993: 36). En este contexto no aparece la relación entre Pachamama e Inti. Para este autor la deidad femenina es asexuada y no requiere de un alter masculino para la reproducción. Por otra parte, la distinción que establece Kessel entre la deidad cristiana y andina no es tan clara. Entender a los uywiris mallku-t'alla como espíritu de los cerros deja fuera dimensiones de la personificación que los aimaras realizan de los seres tutelares. La noción de espíritu oscurece nuestra comprensión sobre las representaciones de las divinidades, pues los contenidos propios del cristianismo eclipsan las ideas aimaras sobre la materialidad intrínseca de los seres vivos (Gavilán et al. 2011).

Irarrázabal (1988: 31) habla de una extendida asociación entre la Virgen cristiana y Pachamama por efecto de la evangelización católica; pero las festividades dedicadas a la Virgen incorporan creencias y ritos propios del culto a la tierra o Pachamama: «María habría sido pachamamaizada y Pachamama marianizada». No obstante, el proceso de apropiación de la cultura judeo-cristiana es más complejo. Existen ámbitos de la vida que parecen ser incompatibles, como lo es la dimensión sexual de las divinidades. Las Iglesias católica y protestante se han esforzado por reprimirla y sancionarla. En tanto que los estudios de la religiosidad andina han tendido a invisibilizarla.

11 En su obra principal, este autor agrega que Pachamama no conoce esposo ni procreador que tuviera relación con ella y su llamada virginidad expresaría explícitamente que ella genera por sí misma y de manera autosuficiente poder creador, la vida en la tierra.
La maternidad como componente central de $\mathrm{Pa}$ chamama es remarcada también por Hans Van den Berg. Él señala que en el altiplano se la imagina como una mujer anciana que durante siglos ha mantenido a sus hijos. Sin embargo, ella es también madre joven, porque sigue siendo capaz de producir frutos (Berg 1989: 289). Kessel (1993: 36) sugiere que los aimaras de las comunidades del altiplano chileno se imaginan a Pachamama como una mujer andina, vestida de aksu que lleva en su Ilijlla toda clase de cultivos y todo tipo de hierbas saludables y medicinales. Imágenes que en nuestras entrevistas corresponderían a mallku-t'alla. Los datos recogidos en las entrevistas aplicadas configuran a Mama Wanapa, montaña protectora de la comunidad de Cariquima, como «una mujer bella, vestida a la usanza antigua, con cara de Inka que sale a caminar en las noches para tener encuentros amorosos con Tata Sillajuay, cerro cercano» (T. M., mujer, 58 años, Cariquima, entrevista personal, [1997]).

Las referencias de Harris (1988) difieren de los autores que enfatizan su carácter maternal inspirado en la Virgen María. Ella afirma que entre los laymi, Bolivia, Pachamama sería la parte femenina de los montes y encarnaría el espacio y el tiempo y sería esposa de las cimas de las montañas. Al mismo tiempo, se relacionaría con los rayos, quien manda los relámpagos. De este modo y como lo indicó Platt (1980), hallamos una vinculación entre Pachamama-montañas y rayos. Harris agrega componentes diabólicos a la divinidad femenina, al caracterizarla como malvada, peligrosa y voraz; por lo cual esta dejaría de tener, los contenidos virginales del catolicismo al destacar también otras dimensiones de la maternidad como el aspecto nutritivo. Ya no sería la madre trascendental, sino más bien representaría el suelo fértil de cuyas cosechas viven los campesinos.

Por su parte, Martínez (2001) señala que las figuras mallku-t'alla serían deidades cuyas manifestaciones ctónicas, demoníacas y benefactoras se ubican en el ukhu pacha o mundo de adentro-abajo. Son identificados con los nombres de diablo, demonio wak'a, saxra o supay. Sugiere que conllevan asociaciones a la fecundidad femenina. Se trataría de algo así como configuraciones uterinas: metales gestándose en las entrañas de la tierra, cavidades uterinas y agujeros creacionales. Afirma que existiría una identidad del Dios del cerro con la Pachamama: «Pachamama no es semióticamente otra cosa que la tematización y la figuración femenina del Diablo-Dios del cerro. Se tra- 
ta de una misma entidad masculino-femenina bisexual o andrógina: qhari-warmi ${ }^{12}$ como dicen en la zona quechua» (Martínez 2001: 139).

La sexualidad como parte del universo religioso es referido por Verónica Salles (1992: 263) en su estudio documental del siglo XVI sobre el encuentro entre el cristianismo con la religión andina en Copacabana. Ella sugiere que la imagen que se venera en Copacabana es una talla de la Virgen de la Candelaria hecha hacia 1582 por Francisco Tito Yupaqui, cuya representación acarreó enfrentamientos entre la visión femenina de los andinos con la europea. Señala que las características de la divinidad femenina autóctona por excelencia, Pachamama y la Virgen María eran tan opuestas que se entraba en contradicciones internas tan profundas e insostenibles que acababa por separarlas. Postula que en la mente de algunos evangelizadores, entre ellos Ramos Gavilán y Arriaga, $\mathrm{Pa}$ chamama y su culto se asociaban no solo con la agricultura y con la fertilidad, sino con la mujer andina y la lascivia. Su referente anciano sería Eva. $P a-$ chamama y los ritos de fertilidad de la mujer, la lascivia y lo demoníaco iban juntos en algunos textos del período. De esta manera, llega a señalar que la asociación Pachamama-concupiscencia hacía imposible su asentamiento o su síntesis con la Virgen María pues los atributos de virginidad castidad y pureza de la Virgen se oponen por exclusión a aquellos de $\mathrm{Pa}$ chamama (Ibid.).

Los datos etnográficos acopiados en este estudio confirman la relevancia de la unidad femenino-masculino en la producción y reproducción de la vida. Lo masculino, representado en Kollanta-Inti o Santísimo alude al padre, es guía de los humanos y protector del orden, pone énfasis en el tiempo (Sol personificado en la figura del Inti). Se ubica arriba, como el rayo y adquiere el carácter de fecundador. Se asocia al aka pacha o con Pachamama en momentos determinados del ciclo anual y productivo, para lo cual requiere de colaboración de sus devotos. Lo masculino se simboliza en las capacidades reproductivas y a su fuerza. Vinculado al tiempo, ordena (o desordena) la vida del presente. Ocupa el lugar principal en el orden de las cosas. En cambio, lo femenino se sintetiza en Pachamama, figura que se desdobla en mallku-t'alla (achachilas o antepasados) en el espacio-territorio de la comunidad; pertenecen al mundo

12 Chacha-warmi en aimara designa la relación esposo-espo- de adentro y vuelven a este mundo en el período comprendido entre Todos los Santos y los carnavales (ver Gavilán y Carrasco 2009).

Estas representaciones condensan las ideas sobre la naturaleza femenina y de las mujeres: procreación, crianza, proveedora. Se simboliza en la producción y reproducción de todos los elementos vitales: sangre, alimentos, agua, música, energía. Pero estas cualidades se activan en su vinculación con lo masculino: son inseparables. Sus potencialidades universales se entienden en el acto de reproducción tal como se manifiesta en la relación esposo-esposa. La castidad, presente en los referentes del cristianismo, no es evidente en la religiosidad aimara. Lo femenino no da vida de manera autónoma, es en la unidad con lo masculino que provee. En la vida diaria, ya sea en el campo o en la ciudad, la presencia de Pachamama se mantiene en la base para nuevas interpretaciones. En su esencialidad se representa como fuente creacional de vida, una especie de configuración uterina que se activa por su sexualidad. Es posible afirmar que el carácter fecundante de Inti o lo de arriba y engendradora de Pachamama, abajo, se inscribe en el campo semántico del deseo sexual; pero ello requiere de nuevos datos. El diccionario de Bertonio (1984 [1612]) indica que la palabra aimara sapaqa significa tanto semilla como semen (Ibid.: 309); y, el término Hatha especifica líneas de descendencia masculina (Ibid.: 124). Llausa, thaltha, vikhira, en cambio, aluden a baba femenina, y, la expresión Llausatha designaría el padecer fluxum feminis, como signo del deseo femenino. De este modo, siendo distintos en sus componentes naturales poseen competencias para la vida como sujetos capaces de crear vida con base en su sexualidad distinguida según el género. Al situar la dimensión sexual en el contexto religioso, la tradición aimara parece valorarla. En cambio, la tradición cristiana hegemónica la ubica en el orden del pecado, lo que contribuye a su ocultamiento.

\section{PALABRAS FINALES: EL GÉNERO COMO SÍMBOLO}

Los antecedentes expuestos muestran procesos de apropiación del cristianismo y no simple imposición. Al comparar los períodos considerados (1982-1997 y 2005-2017) se observa que en los últimos diez años la mayor escolarización de la población promueve cambios en la religiosidad y por lo tanto en los contenidos culturales del género. La descampesinización 
de las nuevas generaciones facilita la adopción de saberes, creencias e ideas modernas, resignificando las concepciones de las diferencias sexuales. Pero, los procesos de translocalización de la comunidad rural a los espacios urbanos implica la vinculación permanente con las localidades de origen y esto se expresa en la producción y reproducción de las fiestas patronales y carnavales. En el contexto del reconocimiento de los pueblos originarios por parte del Estado y la sociedad nacional, se observa que tanto evangélicos como católicos realizan los rituales y producen un discurso que valora el legado de los antepasados. Patrocinar una fiesta de carnaval como Mallku-T'alla implica agradecer a las divinidades, en nombre de su comunidad, por el bienestar económico, salud y armonía familiar; para lo cual se destina una parte importante de los recursos de la unidad familiar. De este modo, se alcanza el mayor prestigio social.

En este escenario, los contenidos culturales asignados a las figuras divinas en términos de género se van aproximando más a los occidentales en un ambiente reflexivo contradictorio. La dimensión sexual del dominio religioso va eclipsándose, pero ello no implica su desaparición. La atención asignada a la relación de pareja y a la fecundación evidencian acciones de sujetos sexuados, pero estas referencias van siendo reemplazadas por la maternidad-paternidad, reduciendo sus cualidades al cuidado y la protección. En este proceso lo femenino va excluyendo su carácter de proveedora de alimentos.

El carácter heterogéneo de las ideas que sustentan la religiosidad aimara puede ser comprendido como unión contradictoria de componentes de naturaleza diferente. La vigencia de las divinidades (pensadas en la dualidad femenino-masculino y en la ambigüedad diabólico/divino) hacen que el devenir de las prácticas religiosas muestre la coexistencia de ideas con bases filosóficas distintas. Esto es visible en el campo de la salud, ya que las concepciones de la naturaleza humana se hallan en la base de las actividades concretas que se deben desplegar para el logro del bienestar de las personas. Vemos como en el año 2005 la muerte de un joven de la comunidad de Isluga y residente en la comuna de Pozo Almonte, afectado por la enfermedad nominada como katjata (agarrado), ratifica la vitalidad de los seres tutelares. Las familias altiplánicas argumentaron que fue devorado por Seren T'alla, deidad de la vertiente de pueblo Isluga a la que recurren principalmente los hombres para inspirarse musicalmente. Se trata de un ser femenino potente a la que se le debe respeto.

El género como símbolo elabora la identidad/alteridad de lo masculino y lo femenino. Las representaciones del sexo binario están presentes como mecanismo para entender las diferencias sexuales que, en su unidad, construyen las bases del orden cósmico y social.

Los contenidos de género esencializan la naturaleza de ambos y la sexualidad constituye un componente central para establecer la diferencia. Este antecedente no coincide con lo señalado por Spedding (1997: 59) pues, aunque la noción de persona (jaque) incluya a ambos géneros, distingue en su interior valores para mujeres y para hombres. Asimismo, aunque las jerarquías están presentes en el interior de lo masculino y de lo femenino, lo primero ocupa el lugar de mayor prestigio. Lo femenino en su esencialidad se representa como fuente creacional de vida, se ubica abajo y adentro, referentes del pasado indígena. Ambas referencias permiten a mujeres y a hombres (en pareja y edad productiva) ordenar e interpretar su mundo social y legitimar sus comportamientos y relaciones. Al mismo tiempo, los símbolos de género presentes en la historia local fundamentan procesos de identidad (pasado indígena) y de alteridad (pasado no indígena).

\section{BIBLIOGRAFÍA CITADA}

Albó, Xavier y Félix Layme. 1992. Literatura Aymara. Antología. La Paz: Centro de Investigación y Promoción del Campesinado, CIPCA/Hisbol/Jayma.

Appadurai, Arjun. 2001. La Modernidad Desbordada. Montevideo/México: Ediciones Trilce/Fondo de Cultura Económica.

Berg, Hans van den. 1989. La Tierra no da así no más: los ritos agrícolas en la religión de los aymara-cristianos de los Andes. Ámsterdam: Centrum voor Studie en Documentatie van Latijns Amerika, CEDLA.

Bertonio, Ludovico. 1984 [1612]. Vocabulario de la Lengua Aymara. Cochabamba: Ediciones del Centro de Estudios de la Realidad Económica y Social, CERES. Disponible en: <https:// archive.org/stream/vocabulariodela00bertgoog\#page/n8/ mode/2up>. Fecha de acceso: 1 ago. 2018.

Bouysse-Cassagne, Térese y Olivia Harris. 1987. «Pacha: en torno al pensamiento Aymara», en Javier Medina, Thérèse Bouysse-Cassagne, Olivia Harris, Tristán Platt y Veronica Cereceda, Tres Reflexiones sobre el Pensamiento Andino: 11-57. La Paz: Hisbol. 
Canessa, Andrew. 1997. «Género, lenguaje y variación en Pocobaya, Bolivia», en Denise Y. Arnold (comp.), Más allá del silencio. Las fronteras de género en los Andes: 233250. La Paz: Centre for Indigenous American Studies and Exchange, CIASE/Instituto de Lengua y Cultura Aymara, ILCA.

Collier, Jane y Michelle Z. Rosaldo. 1981. «Politics and Gender in Simple Societies», en Sherry B. Ortner y Harriet Whitehead (eds.), Sexual Meanings: The Cultural Construction of Gender and Sexuality: 275-329. Cambridge: Cambridge University Press.

Cuche, Denise. 2004. La noción de cultura. Buenos Aires: Nueva Visión.

Esch-Jakob, Juliane. 1994. Sincretismo Religioso de los Indígenas de Bolivia. La Paz: Hisbol.

García Choque, Pedro. 2002 [1997]. Breve diccionario AymaraCastellano, Castellano-Aymara. Aru pirwa. 3.a ed. Iquique: Ediciones Instituto de Estudios Andinos Isluga/ Universidad Arturo Prat.

Gavilán, Vivian. 1998. «Elaboraciones de género en la religiosidad de mujeres y hombres aimara del norte de chile avances de investigación». Revista de Ciencias Sociales 8: 65-82.

Gavilán, Vivian. 2005. «Identidades étnicas en Tarapacá a inicios del siglo XXI». Revista de Dialectología y Tradiciones Populares 60(2): 77-102.

Gavilán, Vivian y A. María Carrasco. 2009. «Festividades y religiosidad andina en el norte chileno». Chungara 1: 101-112.

Gavilán, Vivian, Patricia Vigueras, Michel Parra, Carlos Madariaga, Nicolás Morales, Alejandra Arratia y Rosa Andrade. 2011. "La Sociedad y la Cultura Andina Contemporánea: Estudio de los Saberes para la Salud y la Enfermedad en los Pueblos Originarios del Norte de Chile». Revista de Indias 71(252): 571-600.

Grebe, María Esther. 1981. "Cosmovisión aymara». Revista de Santiago 1: 161-179.

Grebe, María Esther. 1996. "Continuidad y cambio en las representaciones icónicas: significado simbólico sur-andino». Revista Chilena de Antropología 13: 85-93.

Guerrero, Bernardo. 1990. Las campanas del dolor. Violencia y conflicto en los Andes chilenos. Iquique: Ediciones el Jote Errante.

Guerrero, Bernardo. 1994. A Dios Rogando...Los pentecostales en la sociedad aymara del norte grande de Chile. Ámsterdam: Departament of cultural Anthropology/Sociology of Development, Vrije Universiteit.

Gundermann, Hans y Héctor González. 2008. «Pautas de integración regional, migraciones, movilidad y redes sociales en los pueblos indígenas de Chile». Universum 23: 82-115. doi: <https://doi.org/10.4067/S0718-23762008000100006>.

Harris, Olivia. 1980. «The Power of Signs: Gender, Culture and the Wild in the Bolivian Andes», en Carol P. Macormarck y Marilyn Strathern (eds.), Nature, Culture and Gender: 70-94. Cambridge: Cambridge University Press.
Harris, Olivia. 1988. «La Pachamama: significados de la madre en el discurso boliviano», en Mujeres latinoamericanas. Diez ensayos y una historia colectiva: 57-80. Lima: Ed. Flora Tristán, Centro de la Mujer Peruana.

Irarrázaval, Diego. 1988. «Mutación en la identidad andina. Ritos y concepciones de la divinidad». Revista Allpanchis 31: 11-84.

Isbell, Billie J. 1997. "De inmaduro a maduro: lo simbólico femenino y los esquemas andinos de género», en Denise $\mathrm{Y}$. Arnold (comp.), Más allá del silencio. Las fronteras de género en los Andes: 253-298. La Paz: Centre for Indigenous American Studies and Exchange, CIASE/Instituto de Lengua y Cultura Aymara, ILCA.

Kessel, Juan. 1992a. Holocausto al progreso. La Paz: Hisbol.

Kessel, Juan. 1992b. «La Organización Tempo-espacial del Trabajo entre los Aymaras de Tarapacá: la Perspectiva Mitológica», en Silvia Arze y Ana María Lorandi (coords.), Etnicidad economía y simbolismo en los Andes. II Congreso Internacional de Etnohistoria. Coroico: 267-298. La Paz.: Hisbol/ Institut Français d'Études Andines/Sociedad Boliviana de Historia/Antropólogos del Sur Andino.

Kessel, Juan. 1993. Pachamama, La Virgina: la que creó el mundo y fundó el pueblo. Puno: Comisión Internacional de Derechos de Pueblos Indígenas de Sud América, CIDSA.

Kessel, Juan. 2007. "Los andino-cristianos: ¿cristianos o andinos?». Revista Electrónica Volveré 27: 8-16. Disponible en: <http://www.iecta.cl/revistas/volvere_27/articulos. htm\#3a>. Fecha de acceso: 1 ago. 2018.

Lamas, Martha. 1996. "Algunas dificultades en el uso de la categoría género", en Martha Lamas (comp.), El género, la construcción cultural de la diferencia cultural: 327-366. México D. F.: Programa Universitario de Estudios de Género, PUEG.

Martínez, Gabriel. 1976. El sistema de los uywiris en Isluga. Documentos de Trabajo; Publicación n.o 1 marzo. Iquique: Ediciones Internas/Centro Isluga de Investigaciones Andinas.

Martínez, Gabriel. 1989. Espacio y Pensamiento I: Andes Meridionales. La Paz: Hisbol.

Martínez, Gabriel. 2001. «Saxra (diablo) Pachama. La música, tejido, calendario e identidad entre los Jalq'a». Estudios Atacameños 21: 133-152.

Ortiz, Alejandro. 1982. "Moya: espacio, tiempo y sexo en un pueblo andino». Revista Allpanchis 20: 189-208.

Ortner, Sherry y Harriet Whitehead. 1981. «Introduction: Accounting for sexual meanings», en Sherry Ortner y Harriet Whitehead (eds.), The Cultural Construction of Gender: 1-29. Cambridge: Cambridge University Press.

Platt, Tristan. 1980. «Espejos y Maíz. El concepto de yanantín entre los macha de Bolivia», en Enrique Mayer y Ralph Bolton (eds.), Parentesco y matrimonio en los Andes: 139-182. Lima: Pontificia Universidad Católica. 
Platt, Tristan. 1988. "Pensamiento político aymara», en Xavier Albó (comp.), Raíces de América. Mundo Aymara: 355-443. Madrid: Alianza Editorial.

Salles, Verónica. 1992. "Buscando armonía en dos universos religiosos», en Silvia Arce y Ana María Lorandi (coords.), Etnicidad, economía y simbolismos en los Andes. II Congreso Internacional de Etnohistoria Coroico: 255-265. La Paz: Hisbol/Institut Français d'Études Andines/ Sociedad Boliviana de Historia/Antropólogos del Sur Andino.
Spedding, Alison. 1997. «Investigaciones sobre género en Boliva. Un comentario crítico», en Denise Y. Arnold (comp.), Más allá del silencio. Las fronteras de género en los Andes: 53-74. La Paz: Instituto de Lengua y Cultura Aymara, ILCA/ Centre for Indigenous American Studies and Exchange, CIASE.

Strathern, Marilyn. 1979. "Una Perspectiva Antropológica», en Kate Young y Olivia Harris (eds), Antropología y feminismo: 133-152. Barcelona: Anagrama. 